\title{
Faune des eaux souterraines saumâtres de l'Asie Centrale
}

\author{
Par J. A. Birstein et S. I. Ljovuschin ${ }^{1}$ )
}

Avec 3 figures dans le texte

\section{Introduction}

Jusqu'à ces derniers temps les recherches spéléologiques embrassaient presque exclusivement les territoires Sud-Ouest de la partie européenne de l'Union Soviétique. Deux de ses régions - la Crimée et le Caucase - ont été étudiées pendant de longues années, mais la faune souterraine des vastes espaces à l'Est et Sud-Est des Monts Oural n'a jamais été l'objet de recherches spéciales, et nous ne la connaissons que par quelques trouvailles de formes souterraines obtenues accidentellement dans les puits, sources, eaux profondes des lacs. Les géologues et géographes s'occupaient très peu des cavernes de la partie orientale du pays et il n'y a que deux ans qu'on a commencé à récolter leur faune.

Tout ce qui a été dit se rapporte entièrement à l'Asie Centrale. Ses chaînes de montagnes renferment beaucoup de régions karstiques et de grandes cavernes dont certaines sont connues depuis la plus haute antiquité et associées par des légendes aux noms d'Alexandre le Grand, Timur et d'autres héros de l'Antiquité et du Moyen-âge. Quelques trouvailles intéressantes provenant des puits de cette vaste contrée témoignaient de l'originalité de sa faune souterraine.

En 1928 A. L. Brodsky découvrit dans des puits d'eau saumâtre (près de $10 \%$ ) du désert Karakoum plusieurs espèces de Foraminifères, dont la majorité nouvelles pour la science. Il explique cette trouvaille par l'existence sous les sables d'un bassin marin enseveli, resté après le retrait de la mer d'Aktchagyl. Plus tard V. F. Nikoljuk (1948) compléta la liste de Brodsky. Il a observé ces Foraminifères à l'état vivant.

A. I. Jankowskaya (1964) a décrit Microcharon kirghisicus (Crustacea Isopoda) appartenant à un genre d'origine marine, qu'elle découvrit dans les puits des rivages du lac Issyk-Koul (lac sans émissaire superficiel).

\footnotetext{
1) Faculté de Biologie, Université de Moscou.
} 
C'est peut-être la place de mentionner les trouvailles de Bathynellacea dans des puits et des cavernes de l'Asie Centrale à qui plusieurs auteurs sont enclins d'attribuer une origine marine (Delamare Deboutteville, 1960). Nous avons décrit Parabathynella mesasiatica d'un lac cavernicole près de Khaidarkan (Birstein et Ljovuschkin, 1964) et A. I. Jankowskaya (loc. cit.) a découvert ensemble avec Microcharon non seulement Parabathynella, mais encore un autre genre de cet ordre, Bathynella.

Hors de l'U.R.S.S., mais près de sa frontière, en Afghanistan, qui est un prolongement direct de l'Asie Centrale et qui a partagé son destin dans les époques géologiques passées, Bogidiella lindbergi a été récemment trouvée dans une grotte (Ruffo, 1958). Une espèce voisine encore non décrite, appartenant au même genre, a été découverte par nous dans la source Khodja-Kaynar à une distance de $50 \mathrm{~km}$ de la caverne Kaptar-Khana. Comme Microcharon ce genre renferme des formes marines et dulçaquicoles, mais il est beaucoup plus largement répandu (Europe, Afrique du Nord, Israël, Amérique du Sud, île de la Réunion, Petites Antilles).

Donc un trait originel de la faune aquatique souterraine de l'Asie Centrale, qui la distingue de la faune des autres régions de l'U.R.S.S. étudiées sous ce rapport (y compris la Crimée et la Transcaucasie occidentale), est une relative richesse de formes d'origine marine (Foraminifera, Microparasellidae, Bogidiellidae, et, peut-être, Bathynellidae), qui habitent ici les eaux souterraines douces et saumâtres. Pourtant, jusqu'à présent on n'a trouvé dans les différentes régions de l'Asie Centrale que quelques formes isolées d'origine marine. D'autant plus intéressante paraît la découverte de toute une faune souterraine d'eau saumâtre, que nous avons trouvé dans la caverne Kaptar-Khana, située dans les montagnes Kougitang-Tau.

Nous tenons à exprimer notre reconnaissance sincère à tous ceux qui nous ont aidé dans notre travail: le Dr. G. Ch. Dickenstein et ses collaborateurs, qui nous ont fait connaître la géologie et la paléogéographie de la région qui nous intéressait, V. Andreyev, qui mit à notre disposition le plan, levé par lui, de la caverne Kaptar-Khana, le professeur E. W. Borutzky, J. I. Starobogatov, E. M. Mayer, et N. Sumenkova, qui ont aimablement consenti à effectuer la détermination du matériel récolté pour plusieurs groupes et qui nous ont déjà communiqué les résultats préliminaires, aux collaborateurs de la Section de Chimie du Laboratoire des problèmes hydrogéologiques de l'Académie des Sciences de l'U.R.S.S., qui ont fait l'analyse des eaux de la caverne Kaptar-Khana. 


\section{La caverne Kaptar-Khana}

Les montagnes Kougitang-Tau, qui longent la frontière de la Turkménie et de l'Uzbekistan, représentent une des régions karstiques les plus connues de l'Asie Centrale. C'est ici que se trouve la caverne Karlyuk-la plus grande de l'Asie Centrale et une des plus vastes de l'U.R.S.S. Cette caverne est célèbre par ses stalagmites de gypse, ses cristaux et ses fleurs. Plusieurs autres cavernes, moins profondes, sont connues, formées dans les calcaires, gypses et gisements de sel gemme. Au pied des montagnes on rencontre fréquemment des sources karstiques, souvent très puissantes (comme, par exemple, la source KhodjaKaynar).

Comme une prospection spéciale et systématique du karst de cette région n'a pas encore été entreprise, les grottes, même les mieux connues des habitants du pays, n'ont jamais été visitées par des spéléologues. Vers la fin de l'été 1963 un groupe de spéléologues du club de tourisme de Moscou, conduit par V. Andreyev, visita le premier une caverne renfermant un bassin d'eau saumâtre et leva le plan de celleci, que les ouzbeks appellent Kaptar-Khana, ce qui se traduit comme "Maison des pigeons» (fig. 1). Un échantillon de plankton recueilli par V. Andreyev contenait quelques Foraminifères, 2 exemplaires de Harpacticoida et 1 exemplaire de Microcharon (stade manka). En novembre de la même année un de nous (S. L.) visita la caverne pour y effectuer des récoltes plus complètes de cette faune intéressante.

La caverne se trouve sur la rive gauche de la rivière KougitangDarja, à près de $5 \mathrm{~km}$ du coron Mines de plomb, dans le district Khodjambass, région Tchardjou (Turkménie). Elle s'est probablement formée le long de fentes réciproquement perpendiculaires (orientées Est-Ouest et Nord-Sud) dans les gypses datant du Jurassique supérieur. L'accès de cette grotte se fait par un entonnoir karstique, la pente est assez raide, compliquée dans sa partie inférieure par des éboulis et des blocs de gypse effondrés. Sur les saillies de la paroi escarpée méridionale de l'entonnoir nichent des pigeons, qui ont donné leur nom à la caverne. Le fond de l'entonnoir est couvert par des blocs de gypse assez réguliers et plats, séparés par des fentes profondes. Deux galeries partent de l'entrée, l'une orientée Ouest et l'autre Nord-Est. Cette dernière, qui perd bientôt son orientation et tourne Nord-Nord-Ouest, est plus longue (environ 300 mètres), sèche dans la plus grande partie de son parcours et encombrée par les blocs de gypse, recouverts par endroits d'une épaisse couche de guano des Chauves-souris. La galerie Ouest a une longueur de près de 160 mètres. 


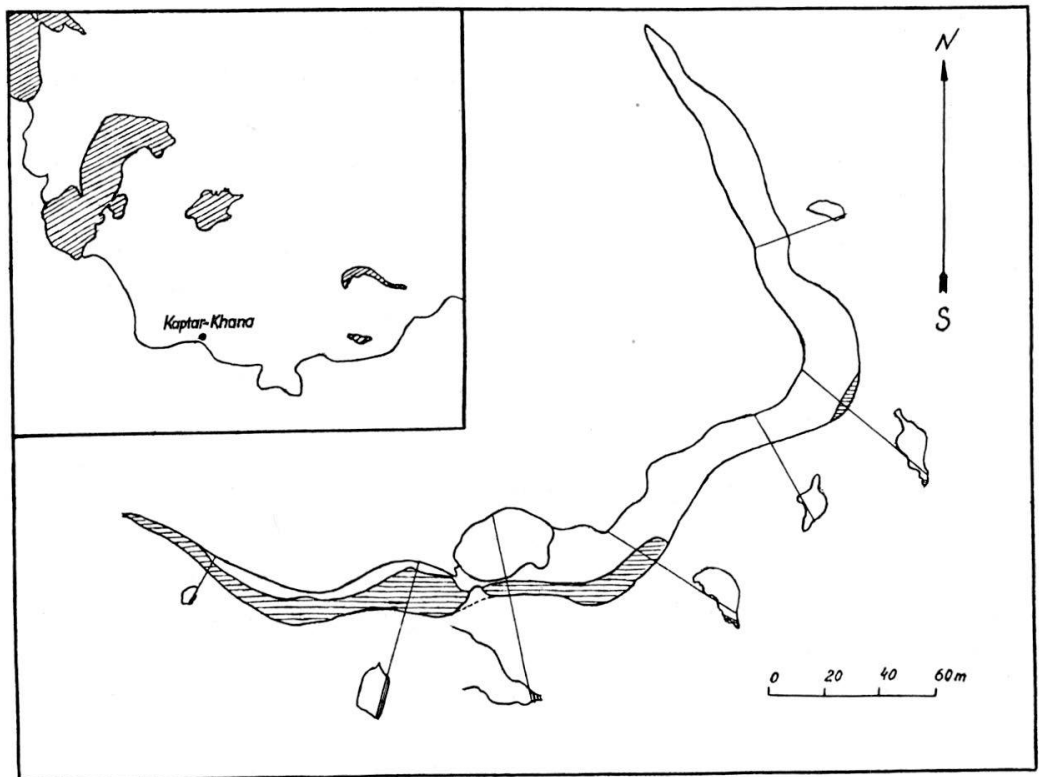

Fig. 1. Plan de la caverne Kaptar-Khana.

La partie Ouest de la caverne renferme un lac, qui disparaît par endroits sous des entassements. Il émerge dans la partie éclairée de la caverne et se termine à l'Est dans la zone demi-obscure. Au tournant de la galerie Nord-Est se trouve un autre lac, beaucoup plus petit, dont la surface est couverte d'une croûte de gypse. La plus grande profondeur du lac atteint 2-2,5 mètres. Vers l'Est elle s'amoindrit et un courant devient visible.

Un trait caractéristique du lac de la caverne Kaptar-Khana est son assez haute salinité $(11,68 \%$ \%o $)$. L'analyse chimique de l'eau est donnée dans le tableau suivant:

Tableau 1

\begin{tabular}{|c|c|c|c|c|c|c|c|}
\hline $\begin{array}{l}\text { Unité } \\
\text { de mesure }\end{array}$ & $\mathrm{Na} \cdot+\mathrm{K} \cdot$ & $\mathrm{Ca} \cdot \cdot$ & $\mathrm{Mg} .$. & $\mathrm{Cl}^{\prime}$ & $\mathrm{SO}_{4}{ }^{\prime \prime}$ & $\mathrm{HCO}_{3}{ }^{\prime}$ & $\Sigma$ \\
\hline $\mathrm{mg} / \mathrm{l}$ & 3412,0 & 675,0 & 136,0 & 5330 & 1835 & 286,5 & 11,68 \\
\hline mg/equiv.-l & 148,4 & 33,7 & 11,2 & 150,4 & 38,2 & 4,7 & 386,6 \\
\hline$\%$ mg/equiv.-l & 38,38 & 8,72 & 2,9 & 38,91 & 9,88 & 1,21 & 100,0 \\
\hline
\end{tabular}


Il est intéressant de comparer la composition en sels de l'eau de la caverne Kaptar-Khana avec celle des eaux de l'Océan Mondial, des mers Caspienne et Aral et du fleuve Amu-Darja, qui se trouve à une distance de $80 \mathrm{~km}$ de la caverne ${ }^{2}$ ):

Tableau 2

\begin{tabular}{|c|c|c|c|c|c|}
\hline $\begin{array}{r}\text { Bassin } \\
\% \mathrm{mg} / \text { equiv. }\end{array}$ & $\begin{array}{c}\text { Lac de } \\
\text { Kaptar- } \\
\text { Khana }\end{array}$ & Océan & $\begin{array}{c}\text { Mer } \\
\text { Caspienne }\end{array}$ & $\begin{array}{c}\text { Mer } \\
\text { d'Aral }\end{array}$ & $\begin{array}{l}\text { Amu- } \\
\text { Darja }\end{array}$ \\
\hline $\mathrm{Na} \cdot+\mathrm{K} \cdot$ & 38,38 & 39,31 & 32,44 & 28,88 & 4,7 \\
\hline $\mathrm{Ca} \cdot \cdot$ & 8,72 & 1,73 & 3,96 & 7,60 & 42,8 \\
\hline Mg.. & 2,9 & 8,95 & 13,78 & 13,68 & 2,5 \\
\hline $\mathrm{Cl}^{\prime}$ & 38,39 & 45,10 & 34,62 & 29,17 & 12,3 \\
\hline $\mathrm{SO}_{4}^{\prime \prime}$ & 9,88 & 44,63 & 14,37 & 19,76 & 15,7 \\
\hline $\mathrm{CO}_{3}^{\prime \prime \prime}+\mathrm{HCO}_{3}^{\prime}$ & 1,21 & 0,20 & 0,83 & 0,91 & 22,0 \\
\hline
\end{tabular}

Comme le montre le tableau, l'eau de Kaptar-Khana diffère beaucoup de l'eau douce de l'Amu-Darja par la proportion des ions et peut être rattachée aux eaux du type marin; en ce qui regarde sa teneur en ions importants $\left(\mathrm{Na} \cdot+\mathrm{K} \cdot \mathrm{Cl}^{\prime}\right)$ elle est plus proche de l'eau océanique que ne le sont les eaux de la mer Caspienne et de la mer d'Aral. Tout de même il n'y a aucune raison d'associer génétiquement l'eau du lac souterrain aux mers anciennes. La composition ionique s'explique parfaitement par les conditions géologiques de cette région, riche en gisements de sel gemme datant du Jurassique supérieur. Il est probable que l'eau du lac de la caverne Kaptar-Khana ait acquis une haute salinité et une composition ionique «marine» en passant par des couches de sel gemme.

\section{Faune}

Dans le bassin d'eau saumâtre de la caverne Kaptar-Khana ont été découverts des Foraminifères, Mollusques, Nématodes, Harpacticoides et Isopodes. Tous les groupes sont représentés partiellement ou totalement par des espèces nouvelles, qui seront décrites ailleurs.

\section{FORA MINIFERA}

Nos récoltes ont été détérminées par E. M. Mayer. D'après ces données elles renforment les formes suivantes:

2) L'océan, les mers Caspienne et Aral d'après L. K. Blinov: «Sur la question de l'origine de la composition saline de l'eau de mer", Meteorol. et Hydrol., N 4, 1947, Amu-Darja d'après O. A. Alekin «Principes de Hydrochimie», 1953. 


\section{Miliammina sp. (fig. 2a)}

Près de 60 ex. à différents stades de croissance. Rappelle une des espèces décrites par Gauthier-Lièvre (1935) du Sahara, mais diffère par une taille moindre et quelques détails de structure. Une autre espèce du même genre est connue, récoltée dans les puits du désert Kara-koum; elle a été determinée comme M.oblonga (Montagu) var. arenacea Chapman (Nikolyuk, 1948), mais cette détermination semble douteuse. Cette forme est presque quatre fois plus petite que la cavernicole, plus multicamérale et possède une dent d'orifice qui manque chez la cavernicole.

\section{Trochamminita sp. (fig. $2 \mathrm{~b}$ )}

Quatre tests vides ont été trouvés, dont deux irrégulièrement enroulés et deux, probablement plus jeunes, planospirales. Cette espèce est très voisine de Tr.irregularis Cushman et Bronimann d'eaux saumâtres du Trinidad (Saunders, 1957).

\section{Borovina zernovi Schmalhausen (?) (fig. 2c)}

Deux tests vides. L'espèce a été décrite du lac Balpasch-sor (Kazakhstan) d'après des tests vides (Schmalhausen, 1950). Selon O. I. Schmalhausen «le plus probable est que le Foraminifère de Balpasch habite les eaux interstitielles et, entrainé par elles dans le lac, périt dans les conditions de minéralisation excessive». P. D. Resvoy (1951) trouva dans le lac Bolchoy Goumikovsky (lit délaissé de la rivière Vakhch) deux tests vides de Foraminifères qu'il attribue à titre de supposition à cette espèce. Une forme semblable sous des noms différents, correspondant à différents stades de développement, a été décrite d'après des tests vides par V. Krapin (1929) du sol de petites rivières qui se jettent dans le lac Elton. Il est donc possible que B.zernovi soit largement répandue dans les eaux interstitielles saumâtres de l'Asie Centrale.

Cette espèce est proche de Jadammina polystoma Bartenstein connue d'eaux saumâtres du littoral de l'Allemagne et de la Hollande (Bartenstein und Brand, 1938) et appartient probablement au même genre. B.zernovi se distingue d'Entzia tetrastomella Daday, propre aux étangs salés de la Transylvanie, par la forme de l'orifice mais lui ressemble par sa taille et la structure du test chitineux-arénacé.

\section{NÉMATODES}

La forme découverte dans la caverne appartient à la famille Oncholaimidae (det. N. Soumenkova) et est apparentée aux espèces marines 
et d'eaux saumâtres qui y dominent. Surtout elle est proche d'Oncholaimus et d'Eurystamina, qui se remontrent fréquemment dans les eaux interstitielles marines.

\section{MOLLUSQUES}

Le Gastéropode déterminé par J. I. Starobogatov est très proche de «Caspia» issykkulensis Clessin et mérite qu'un genre spécial soit institué pour l'y inclure avec cette dernière: Pseudocaspia Starobogatov gen.n. (Truncatellidae Pomatiopsinae). Dans la caverne KaptarKhana habite une nouvelle espèce de ce genre-P.ljosuschkini (fig. $2 \mathrm{~d}$ ),

A
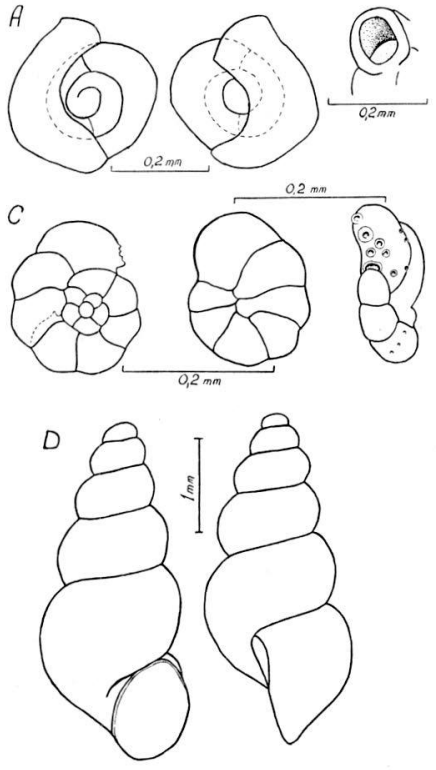
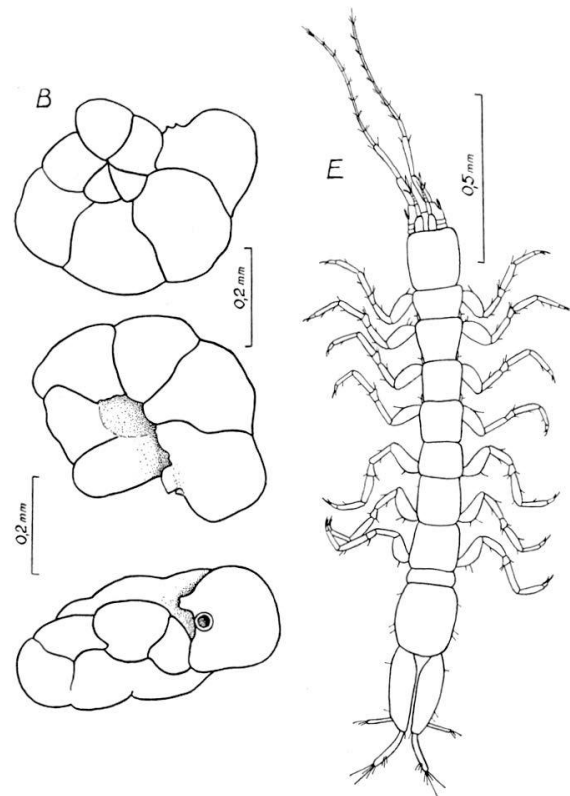

Fig. 2. Quelques représentants de la faune de la caverne Kaptar-Khana:

$\mathrm{A}=$ Miliammina sp.

$\mathrm{B}=$ Trochamminita $\mathrm{sp}$.

$\mathrm{C}=$ Borovina zernovi Schm. (?)
$\mathrm{D}=$ Pseudocaspia ljosuschkini sp.n.

$\mathrm{E}=$ Microcharon halophilus sp.n.

qui se distingue de l'espèce type par des sphères plus saillantes, une coquille moins svelte, une fente ombilicale bien marquée et un angle plus arrondi au bord supérieur de l'orifice. 


\section{COPÉPODES HARPACTICOIDES}

E. W. Borutzky a découvert dans nos récoltes trois espèces, appartenant aux genres suivants:

\section{Ectinosoma sp.}

Ce genre marin comporte d'après Lang (1948) vingt-sept espèces incontestables et trois espèces signalées mais pas décrites (de la mer Caspienne). La plupart de ces espèces (21) habitent la partie septentrionale de l'Océan Atlantique et l'Océan Arctique; deux espèces ( $E$. melaniceps Boeck et E. herdmanni T. et A. Scott) sont plus largement répandues, deux sont connues de l'Antarctique, une du port d'Alexandrie, une des eaux côtières de l'Afrique, deux des bassins salés du littoral de la France (Le Croisic) et trois ou quatre espèces ont été signalées pour la mer Caspienne. Il n'y a qu'une espèce de ce genre l'E. abrau (Kritschagin) - qui habite non seulement les eaux saumâtres mais aussi les eaux douces, en particulier la partie aval de l'AmuDarja, mais d'après une communication de E. W. Borutzky cette espèce est éloignée de l'espèce cavernicole de Kaptar-Khana.

Certaines espèces d'Ectinosoma peuvent exister dans les eaux interstitielles marines: E.curticorne Boeck a été signalée pour le littoral de la Finlande (Delamare, 1960) et E.reducum Bozic a été décrite des eaux interstitielles marines de Roscoff (Bozic, 1955).

\section{Schizopera paradoxa (Daday)}

Cette espèce a été découvert dans le lac Issyk-Kul et plus tard se retrouva dans les bassins des lacs Elton et Baskountchak, dans les eaux saumâtres partout (Borutzky, 1952).

Cl. Delamare Deboutteville (1960) dans son excellent livre donne une liste des espèces de genre Schizopera et une analyse de leur distribution. Il arrive aux conclusions suivantes: «Le genre possède en Europe son maximum de diversification dans les eaux souterraines littorales... Par contre, s'il peut peupler parfois les eaux douces de grottes des systèmes karstiques littoraux en Europe il ne s'étend jamais dans les eaux douces continentales de surface» (p. 622).

Il faut noter la présence de quatre espèces endémiques dans la mer Caspienne (Sars, 1927; Akatova, 1935). En ce qui regarde les espèces endémiques dans les lacs Tanganjika, Victoria et Victoria-Nyanza, 
Delamare Deboutteville admet la possibilité de leur pénétration dans ces lacs grâce aux transgressions marines tertiaires.

\section{Nitocra sp.}

D'après les données de E. W. Borutzky (1952) le genre Nitocra «renferme onze espèces qui présentent des transitions complètes du mode de vie maritime au dulçaquicole; en masse les représentants de ce genre sont des habitants d'eaux saumâtres et sont largement répandus» (p. 122). Un certain nombre d'espèces ont été découvertes dans les eaux interstitielles des côtes maritimes (N.spinipes Boeck-en Allemagne et Jugoslavie, N.lacustris [Schmank.] - en Finlande, $N$. pontica Jakub. - en Roumanie, $N$. cari Petk., N. typica adriatica Petk. et $N$. affinis rijkana Petk. - en Jugoslavie). La découverte la plus proche de notre emplacement est celle de N.lacustris [Schmank.] dans les lacs Elton, Baskounchak et dans le delta d'Amu-Darja.

\section{ISO PODES}

Microcharon halophilus sp. n. (fig. 2e)

Dix femelles et un mâle ont été découverts d'une longueur de 1,25-1,40 mm. La description de cette nouvelle espèce est publiée ailleurs.

Le genre Microcharon Karaman renferme quatorze espèces (y compris $M$. halophilus et $M$. tantalus que nous avons découvert sur le littoral caucasien de la mer Noire), cinq variétés et deux formes indéterminées jusqu'à l'espèce. Deux d'entre elles, les espèces marines $M$. teissieri (Lévi) et $M$. harrisi Spooner, habitent dans la Manche, douze autres et les deux formes indéterminées peuplent les eaux interstitielles saumâtres et douces des bassins de la Méditerranée et de la mer Noire et deux espèces de l'Asie Centrale habitent l'une dans les eaux interstitielles du bassin du lac Issyk-Koul (Jankowskaya, 1964) et l'autre la caverne Kaptar-Khana (fig. 3).

L'origine marine du genre Microcharon est universellement reconnue. Spooner (1959) admet même une répartition cosmopolite de ce genre dans l'Océan Mondial. D'autre part, Chappuis et Delamare Deboutteville (1960) attribuent à Microcharon une origine méditerranéenne. Ces auteurs tiennent pour des relictes des mers tertiaires les espèces découvertes à une distance considérable des côtes de la mer. 


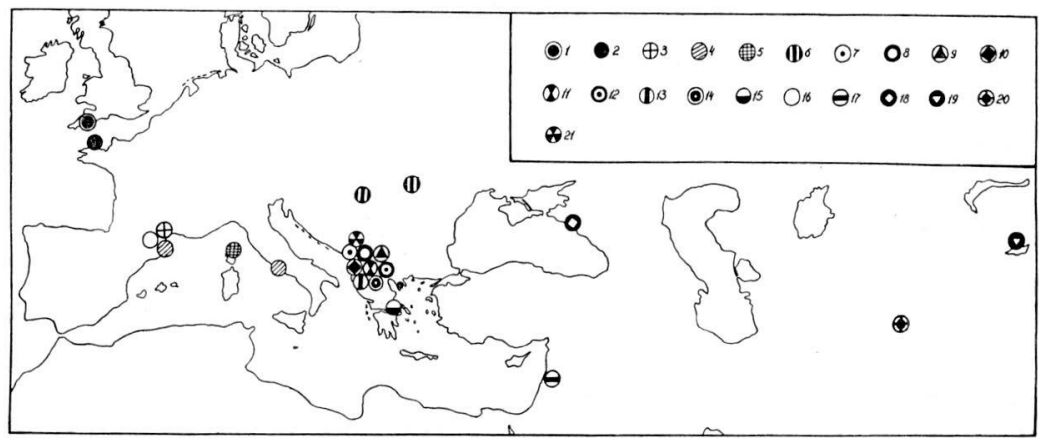

Fig. 3. Distribution géographique du genre Microcharon (d'après Delamare Deboutteville, avec des compléments).

$1=M$. harrisi Spooner

$2=M$. teissieri (Lévi)

$3=M$. angelieri Coineau

$4=M$. marinus Chapp. et Del.

$5=M$. sisyphus Chapp. et Del.

$6=M$. acherontis Chapp.

$7=$ M. latus Kar.

$8=M . l$. prespensis Kar.

$9=$ M. stygius Kar.

$10=M$. profundalis Kar.

$11=$ M. pr. kumanovensis Kar.
$12=M$. pr. kosovensis Kar.

$13=M$. pr. beranensis Kar.

$14=M$. major Kar.

$15=$ M. st. hellenae Chapp. et Del.

$16=M$. sp.

$17=M . \mathrm{sp}$.

$18=M$. tantalus Birst. et Ljov.

$19=$ M. kirghisicus Jank.

$20=M$. halophilus Birst. et Ljov.

$21=$ M. hercegooinensis Kar.

\section{La question de l'origine de la faune de Kaptar-Khana}

Tout ce qui a été dit indique que la faune d'eau saumâtre découverte dans la caverne Kaptar-Khana est manifestement apparentée à la faune du bassin méditerranéen et du bassin ponto-caspien ${ }^{3}$ ). Et ce n'est pas un hazard si d'une part une affinité existe entre les Foraminifères plus nombreux et sans aucun doute vivants du genre Miliammina propres à la caverne Kaptar-Khana et les des eaux interstitielles du Sahara, que d'autre part les Harpacticoides de cette caverne appartiennent à des genres constitués essentiellement par des espèces européennes et qu'enfin Microcharon soit apparenté aux espèces circummediterranéennes de ce genre. La faune du bassin cavernicole de Kaptar-Khana semble être un fragment rejetté très loin vers l'orient de la faune interstitielle circum-méditerranéenne. Toute tentative

3) Excepté Pseudocaspia ljosuschkini; J. I. Starobogatov (in litt.) tient le genre Pseudocaspia pour un élément de l'Asie Orientale dans la faune de l'Asie Centrale. 
d'expliquer son existence doit partir des transgressions marines qui s'approchaient de l'Ouest vers la région en question.

Le dernier bassin marin qui inondait directement le territoire où se trouve la caverne, existait durant tout le Paléogène. C'était une mer peu profonde, dont la salinité a été sujette à de variations considérables (ainsi ce bassin a été déssalé au Paléocène supérieur). Avec l'Oligocène commence le retrait de la mer, de vastes lagunes sont formées et un régime continental s'établit.

Le début du Néogène est caractérisé par des mouvements orogénétiques puissants. Les oscillations verticales atteignent une amplitude de 12-14 km. Au cours du Pliocène la transgression de la mer d'Akchagyl s'étend de nouveau vers l'Orient, mais les montagnes Kougitang-Tau demeurent terre ferme. Les dépôts d'Akchagyl les plus proches de Kaptar-Khana ont été découverts aux environs du bourg Zakhmet (région Mary), à une distance de $300 \mathrm{~km}$. de la caverne, mais il est très probable que la mer s'étendait encore plus loin, pour le moins jusqu'à l'Ouzboy de Keliff (à $120 \mathrm{~km}$. de la caverne) (Dickenstein, 1964).

A laquelle de ces transgressions faut-il rapporter la faune d'eau saumâtre de Kaptar-Khana? On pourrait la considérer comme une relicte de la mer du paléogène, mais les mouvements tectoniques puissants ont provoqué des bouleversements catastrophiques de la structure orographique et hydrographique de la région et il est douteux que des formes relativement sténothermes aient pu subsister lors des déplacements abrupts verticaux, accompagnés de brusques changements de température. Peut-être faut-il tenir les habitants de KaptarKhana pour des descendants des habitants de la mer d'Akchagyl. On sait que certains animaux, qui quittent le littoral marin pour venir habiter le système d'eaux douces interstitielles, sont capables de se répandre activement sur des dizaines de $\mathrm{km}$. vers le fond des continents. Des données pareilles existent pour Microcharon angelieri et $M$.acherontis. On peut admettre que $M$.halophilus aussi s'est répandu par le système d'eaux interstitielles (peut-être saumâtres) jusqu'à son aire d'habitation actuelle. Mais il est plus difficile d'accepter cette hypothèse pour les Foraminifères.

Nous ne pouvons donc considérer la faune de Kaptar-Khana comme une relicte des mers du Paléogène et, en même temps, comme une relicte de la mer d'Akchagyl sans nous heurter à nombre de difficultés. Il n'a pas été possible jusqu'ici de trouver une explication satisfaisante de la découverte de formes marines dans la caverne de la crête Kougitang-Tau. Encore moins compréhensible est la présence de relictes marins dans certaines autres eaux souterraines de l'Asie Centrale, 
comme en particulier celle de $M$. kirghisicus dans la région du lac Issyk-Koul. Le Thian-Chan septentrional est devenu un territoire d'érosion déja durant le Carbonifère supérieur et son régime continental demeura invariable tout le temps suivant. Il est vrai que la mer paléogène entourait le Thian-Chan du Nord et du Sud, mais les dépôts marins de l'Eocène et de l'Oligocène supérieur les plus proches sont connus à une distance d'environ $500 \mathrm{~km}$. du lac, dans la vallée de Tchou (Petrouchevsky, 1948; Ranzman, 1959).

Et s'il est encore possible de dater le peuplement de la région de la crête Kougitang-tau par le genre Microcharon du Pliocène, la date du peuplement par ce genre de la région d'Issyk-Koul doit être reculée non seulement au delà du Néogène mais de toute la période tertiaire. Cela témoigne d'une très grande ancienneté de ce genre et permet de supposer qu'il existait déjà dans les eaux interstitielles littorales de mers plus anciennes de l'Asie Centrale.

\section{RÉSUMÉ}

Dans la grotte Kaptar-Khana (Sud-Ouest de la Turkménie) a été découvert un lac dont l'eau présente une salinité de $11,68 \%$.

Ce lac est habité par une faune d'origine marine: Foraminifères (trois espèces), Mollusques (Pseudocaspia ljovuschkini sp.n.), Harpacticoida (genera Ectinosoma, Schizopera et Nitocra); Isopodes (Microcharon halophilus sp.n.) et peut-être des Nématodes (Oncholaimidae). La majorité des espèces découvertes sont connues commes des espèces d'origine circumméditerranéenne.

La datation géologique ne permet pas de considérer cette faune comme une relicte de l'une des mers tertiaires. Ce même fait se reproduit dans tous les autres cas où des animaux d'origine marine ont été découverts dans des eaux souterraines de l'Asie Centrale (comme par exemple Microcharon kirghisicus Jank. des rives du lac Issyk-Kul). Nous pouvons donc soit admettre une ancienneté beaucoup plus importante de cette faune, soit la possibilité que les formes qui la composent se soient dispersées très largement au delà des limites des transgressions marines.

\section{SUMMARY}

In the cave Kaptar-Khana (south-western Turkmenia) was discovered a lake filled with water with a salinity of $11,68 \%$.

This lake is inhabited by a fauna of marine origin - Foraminifera (three species), Molluses (Pseudocaspia ljovuschkini sp.n.), Harpacticoida (genera Ectinosoma, Schizopera and Nitocra), Isopoda (Microcharon halophilus sp.n.) and possibly Nematoda (Oncholaimidae). The majority of the discovered species are related to species of circum-mediterranean origin.

Geological data do not permit to consider this fauna as a relict of any of the tertiary seas. The same applies to all other cases when animals of marine origin were discovered in subterranean waters of Central Asia (as for instance Microcharon kirghisicus Jank. on the shores of the lake Issyk-Kul). 
We can either admit a far greater anciennety of this fauna or an ability of its components to disperse very widely beyond the boundaries of marine transgressions.

\section{BIBLIOGRAPHIE}

Aкatowa, N. (1935) - Drei neue Copepoden-Arten aus dem Kaspi-See. Zool. Anz., Bd. III, H. 11/12: 319-326.

Bartenstein, H., und Brand, E. (1938) - Die Foraminiferen-Fauna des Jade-Gebietes. Senckenbergiana, Bd. 20, $\mathrm{N}^{\circ} 5: 381-386$.

Birstein, J. A., and Ljovuschkin, S. I. (1964)-Occurence of Bathynellacea (Crustacea Syncarida) in Central Asia. Zool. Journ., Vol. XLIII, N 1 : 17-27 (en russe avec res. angl.).

- - (1965) - The subterranean Paraselloidea (Crustacea Isopoda) in U.S.S.R. Zool. Journ. (sous presse).

Borutzky, E. W. (1952) - Harpacticoida des eaux douces. Faune de l'U.R. S.S., Vol. III, N 4: 1-425 (en russe).

Bozic, Br. (1955) - Copépodes Harpacticoides des sables des environs de Roscoff. Arch. zool. expér. gén., Vol. XCII, N 1: 1-12.

Brodsky, A. L. (1928) - Foraminifera (Polythalamia) in the wells of the Kara-Kum desert. Trans. Centr. Asiat. State Univers. Tashkent, Vol. VIII, N. 5: 1-16 (en russe).

Chappuis, P. A., et Delamare Deboutteville, Cl. (1960) - États de nos connaissances sur une famille et une sous-famille: les Microparasellidae et Microcerberinae (Isopodes) des eaux souterraines. In: Cl. Delamare, 1960 : 293-357.

Delamare Deboutteville, Ci. (1960) - Biologie des eaux souterraines littorales et continentales. Paris, 1-740.

Dickenstein, G. Сh. (edit.) (1964) - Les conditions de la sédimentation et la caractéristique géochimique des dépôts meso-cainozoiques des régions occidentales de l'Asie Centrale. Moscou (en russe).

Gauthier-Lièvre, L. (1935) - Sur une des singularités des l'oued Rhir: des Foraminifères Thalassoides vivants dans les eaux sahariennes. Bull. Soc. H. N. Afr. Nord., Vol. XXVI : 142-147.

JANKOWSKAJA, A. I. (1964) - Relict crustaceans of coastal bottom waters of the lake Issyk-Kul (North Tien-Shan). Zool. Journ., Vol. XLIII, N 7: 975-986.

Krapin, V. (1929) - Über die Foraminiferen der in den Eltonsee einmündenden Flüsse. Russ. Hydrobiol. Zeitschr., Bd. VIII, N 6-7: 187-192 (en russe avec res. allemand).

LANG, K. (1948) - Monographie der Harpacticiden. Stockholm, 1-1683.

NikolJUK, W. F. (1948) - The relict Foraminifera of the Kara-Kum desert. Bull. Ac. Sci. Uzbek. S.S.R., Vol. 1: 79-88 (en russe).

Petrouchevsix, B. A. (1948) - La structure des dépôts tertiaires de Thian-Chan. Bull. Soc. nat. Moscou, sér. géol., Vol. 23, N 1: 53-91 (en russe).

- (1948) - Sur l'histoire de développement de Thian-Chan pendant les temps méso- et cainozoique. Bull. Soc. nat. Moscou, sér. géol., Vol.23, N 5: 55-71 (en russe).

Ranzman, E. J. (1959) - Géomorphologie de vallon de lac Issyk-Koul et de leur encadrement de montagne. Moscou, 1-88 (en russe). 
Rezvoy, P. D. (1951) - The occurrence of the Foraminifera in the lake Greath Gulikovsky. C. R. Ac. Sci. Tadjik. S. S. R., Vol. 1: 43-46 (en russe).

Rufro, S. (1958) - Due nuove specie di Anfipodi delle acque sotteranee dell'Afghanistan. Mem. Mus. Civ. St. Nat. Verona, Vol. VI: 389-403.

SArs, G. O. (1927) - Notes on the Crustacean fauna of the Caspian Sea. Festschrift Prof. N. M. Knipovitch, 315-329.

Saunders, J. B. (1957) - Trochamminidae and certain Lituolidae (Foraminifera) from the recent brackish-water sediments of Trinidad, British West Indies. Smiths. Misc. Coll., Vol. 134, N 5: 1-16.

Schmalhausen, O. (1950) - The new species of Foraminifera from the lake Balpash-Sor (Kazachstan). C. R. Ac. Sci. U.R.S.S., Vol. 75, N 6 : 869-872 (en russe).

Spooner, G. M. (1959) - The occurence of Microcharon in the Plymouth offshore bottom fauna, with description of a new species. Journ. Mar. Biol. Assoc. U. K., Vol. 38, N 1: 57-63. 